\title{
Heavy Quark Coupled Channel Dynamics from Thermal Shifts
}

\author{
Enrique Ruiz Arriola ${ }^{1, a}$ b , Lorenzo Luis Salcedo ${ }^{1}$, and Eugenio Megías ${ }^{2,3}$ \\ ${ }^{1}$ Departamento de Física Atómica, Molecular y Nuclear and Instituto Carlos I de Física Teórica y Computa- \\ cional. Universidad de Granada, E-18071 Granada, Spain. \\ ${ }^{2}$ Max-Planck-Institut für Physik (Werner-Heisenberg-Institut), Föhringer Ring 6, D-80805 Munich, Germany \\ ${ }^{3}$ Departamento de Física Teórica, Universidad del País Vasco UPV/EHU, Apartado 644, 48080 Bilbao, Spain
}

\begin{abstract}
QCD at finite temperature below the phase transition should be determined in terms of colour singlet states such as hadrons and strings. We show how quark-hadron duality allows extracting sensible information concerning heavy quark and string breaking coupled channel dynamics from Polyakov loop correlators.
\end{abstract}

\section{Introduction}

A general principle of quantum statistical mechanics is the direct relation between the thermodynamics of the system at equilibrium at finite temperature $T$ and the spectrum of all possible states $\Psi_{n}$ of a given Hamiltonian $H$, fulfilling $H \Psi_{n}=E_{n} \Psi_{n}$ [1]. In a finite box with volume $V$ all states are discretized. Actually, the partition function counts every single state with a Boltzmann factor,

$$
Z=\operatorname{Tr} e^{-H / T}=\sum_{n} e^{-E_{n} / T} \equiv e^{-F / T},
$$

where the Helmholtz free energy $F$ has been introduced. As the Hamitonian itself, $F(T, V)$ is determined up to an arbitrary constant which may be fixed by a reference temperature, say $F_{0}=F\left(T_{0}, V\right)$. All thermodynamical observables of the system may be deduced by the standard relations for the entropy $S$ or the physically measurable specific heat $c$ (which we rewrite as a fluctuation [1]),

$$
S=-\partial_{T} F, \quad c=T \partial_{T} S=\partial_{T} U=(\Delta H)^{2} / T^{2}>0,
$$

where $(\Delta H)^{2}=\left\langle H^{2}\right\rangle_{T}-\langle H\rangle_{T}^{2}$. In the thermodynamic limit, $V \rightarrow \infty$, we expect the volume to factorize $F \rightarrow-V p, S \rightarrow V s, E=V \epsilon$. However, when we add test particles $\left(Q_{1}, \ldots, Q_{N}\right)$ to the system, the Hamiltonian and its eigenvalues are modified, $H \rightarrow H+\sum_{i=1}^{N} V_{Q_{i}}, E_{n} \rightarrow E_{n}+\Delta E_{n, Q}$, and there appears a volume independent shift of the thermodynamic quantities,

$$
\Delta F \equiv F_{Q_{1} \ldots Q_{N}}, \quad \Delta S \equiv S_{Q_{1} \ldots Q_{N}}, \quad \Delta c \equiv c_{Q_{1} \ldots Q_{N}} .
$$

\footnotetext{
ae-mail: earriola@ugr.es

${ }^{b}$ Presenter at XIIth Conference on Quark Confinement and the Hadron Spectrum, August 29th to September 3rd 2016, Thessaloniki, Greece. E.R.A. thanks D.R. Entem for discussions. This work is supported by Spanish Ministerio de Economía y Competitividad and European FEDER funds under contracts FIS2014-59386-P and FPA2015-64041-C2-1-P, Junta de Andalucía grant FQM-225, and Spanish Consolider Ingenio 2010 Programme CPAN (CSD2007-00042). The research of E.M. is supported by the European Union under a Marie Curie Intra-European fellowship (FP7-PEOPLE-2013-IEF) with project number PIEF-GA-2013-623006, and by the Universidad del País Vasco UPV/EHU, Bilbao, Spain, as a Visiting Professor.
} 
In this contribution we review how these thermodynamic shifts provide information on heavy quark string breaking dynamics when a test heavy quark-antiquark pair separated at a fixed distance is placed in the hot QCD vacuum below the phase transition [2-6]. Note the these shifts are not true thermodynamic quantities. For instance $c_{Q}=\Delta c$ is the difference of two positive contributions but not necessarily positive itself ${ }^{1}$.

\section{The hot QCD vacuum and the hadronic spectrum with u,d,s quarks}

Because of confinement the QCD spectrum is made of colour singlet states. Up to electroweak corrections, we identify it with the hadronic spectrum in the case of bound states. This identification becomes a problematic issue in the case of a resonance where mass and width depend on the particular production process and the corresponding background definition in the experiment whereas in a finite box such as in lattice QCD, a resonance is identified as a volume independent energy shift ${ }^{2}$. The question turns critical when the number of states becomes large as it happens at finite temperature. Particle states are termed hadrons when they are either bound states or resonances and are phenomenologically listed in the PDG [8]. So far, the current 2016 classification echoes the naive quark model where mesons are $\bar{q} q$ and baryons are $q q q$ states; the remaining "further states" [8] await consensual identification and will be disregarded here. Therefore, it makes sense to compare with the Relativized Quark Model (RQM) spectrum computed by Isgur, Godfrey and Capstick in 1985 [9, 10]. We illustrate a comparison between the 2016 PDG and the 1985 RQM in Fig. 1. As can be seen, both spectra are different, but it will be shown that they are thermodynamically equivalent.

The thermodynamic approach provides a more global and balanced comparison adapted to the physical situation such as e.g. ultrarelativistic heavy-ions collisions where the equation of state (EOS) plays a role in the hydrodynamics and kinetics of the process [11]. From the QCD side it is known that there exists a smooth crossover [12] at about $T_{c}=150 \mathrm{MeV}$ (and zero chemical potential) which can be characterized by a maximal violation of scale invariance of the trace anomaly $\mathcal{A}(T)=(\epsilon-3 p) / T^{4}(\mathcal{A}(T)$ vanishes for massless particles). The lattice calculations by the Bielefeld and Wuppertal groups $[13,14]$ comprise decades of efforts which merging results are summarized in Fig. 1 for $120 \mathrm{MeV} \leq T \leq 300 \mathrm{MeV}$.

On the phenomenological hadronic side, one may assume that the EOS is determined by hadron dynamics, where all resonance states which live long enough contribute to the thermodynamics. This is unlike some weakly bound states, such as e.g. the deuteron, which average out within temperature intervals comparable to the binding energy [3]. The Hadron Resonance Gas (HRG) implements this idea as a multicomponent system of non-interacting pointlike hadrons which is expected to work below $T_{c}$. If $M_{n}$ are the masses of the hadrons one has

$$
\mathcal{A}(T)=\frac{\epsilon-3 p}{T^{4}}=\sum_{n} g_{n} \int \frac{d^{3} p}{(2 \pi)^{3}} \frac{E_{n}(p)-p \cdot \nabla_{p} E_{n}(p)}{e^{E_{n}(p) / T}+\eta_{n}},
$$

where $g_{n}$ is the degeneracy, $E_{n}(p)=\sqrt{p^{2}+M_{n}^{2}}$ and $\eta_{n}=\mp 1$ for Bosons and Fermions respectively. In Fig. 1 we compare the HRG when the spectrum is made out of the states listed in the PDG [8] or taken from the RQM $[9,10]$ with the recent lattice calculations [13, 14]. This overall agreement between the venerable RQM-based HRG, the upgraded PDG-based HRG and the brand new QCD lattice calculations is truly impressive, although not fully understood.

\footnotetext{
${ }^{1} \mathrm{~A}$ familiar example is given by the lowering of specific heat of snow in the presence of common salt $(\mathrm{NaCl})$ which melts the mixture, meaning that the entropy shift decreases with temperature. This effect is actually seen in QCD where $\Delta c \equiv c_{Q}<0$ above the critical temperature [5]. Renormalization group equations for these quantities have been elaborated [6].

${ }^{2}$ It only becomes unambiguous as pole in the complex plane, what requires analytical continuation to the second Riemann sheet since complex energies are not directly measurable. This introduces a half-width ambiguity in the resonance mass [7].
} 

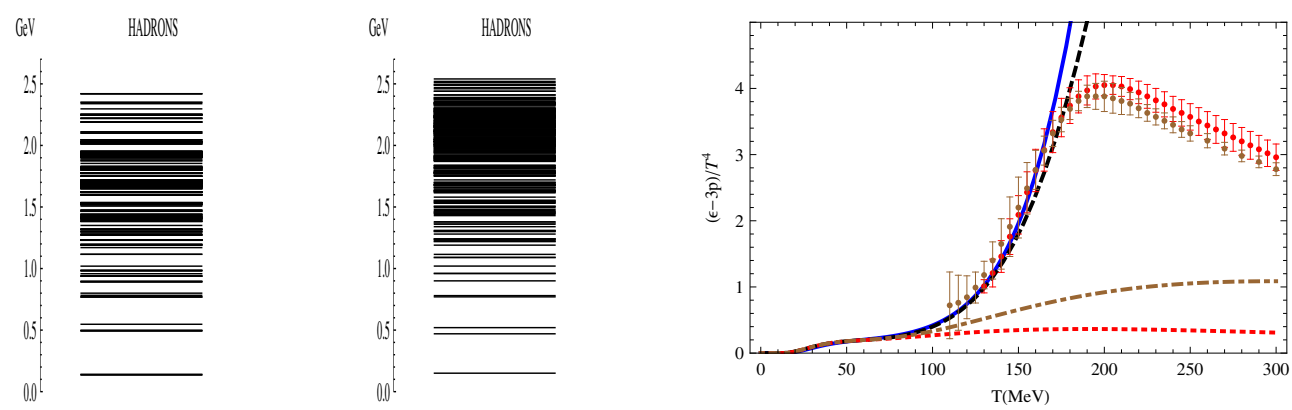

Figure 1. Left panel: The PDG hadron spectrum [8] Middle panel: The RQM hadron spectrum [9, 10]. Right panel: Trace Anomaly in lattice QCD $[13,14]$ vs HRG using PDG (dashed) and RQM (solid) spectra. We also plot just the contribution of states with $M<0.6 \mathrm{GeV}$ (dotted) and $M<0.8 \mathrm{GeV}$ (dotted-dashed).

\section{Heavy quarks and quarkonia}

Heavy quark phenomenology is guided by abundant and varied information on heavy quarkonia spectra and electroweak as well as strong decays. Besides, their large mass allows to work in the BornOppenheimer approximation and to treat their low-lying bound states nonrelativistically. The Cornell potential works phenomenologically well for hidden- charm and bottom $\bar{c} c$ and $\bar{b} b$ bound state mesons such as $\psi(n S)$ or $\Upsilon(n S)$ respectively. This potential has also been reproduced on the lattice as an static energy accurately resembling that of a bosonic string of length $r$ in the ground state [15]

$$
V_{\bar{Q} Q}(r)=\lim _{m_{\bar{Q}}, m_{Q} \rightarrow \infty}\left[E_{\bar{Q} Q}(r)-m_{Q}-m_{\bar{Q}}\right]=-\frac{\pi}{12 r}+\sigma r+c .
$$

The constant $c$ is ambiguous and depends on the renormalization scheme. A renormalization prescription consists of imposing that the $\Upsilon(1 S)$ state wave function be node-less, in agreement with the oscillation theorem (see e.g. [16]). Phenomenologically it takes the common value $c=-250 \mathrm{MeV}$ when $\sigma=(0.42 \mathrm{GeV})^{2}$ in the RQM $[9,10]$ leading also to the $u, d, s$ spectrum of Fig. 1 as well as charmonium and bottomonium spectra.

Of course, some $\bar{Q} Q$ quarkonium states are unstable and undergo a strong decay into heavy-light mesons $B=\bar{q} Q$ and anti-mesons $\bar{B}=\bar{Q} q$ and can thus be interpreted as bound states in the continuum, such as $\Upsilon\left(4^{3} S_{1}\right) \rightarrow \bar{B} B \equiv(\bar{b} u)(\bar{u} b)$ (see Ref. [17] for a RQM perspective within the ${ }^{3} P_{0}$ model ). The underlying mechanism is attributed to string breaking between the colour conjugated sources generating energetically favoured light $\bar{q} q$ pairs from the vacuum and forming weakly interacting colour neutral states. In the Born-Oppenheimer approximation this happens when the corresponding energies cross $E_{\bar{Q} Q}\left(r_{c}\right)=M_{\bar{q} Q}+M_{q \bar{Q}}$ and there is a transition potential $W(r) \equiv V_{\bar{Q} Q \leftrightarrow \bar{B} ; \bar{B}}(r)$.

Quite generally this yields a multiple avoided crossing pattern, among $B_{n}=(\bar{q} Q)_{n}$ and $\bar{B}_{m}=(\bar{Q} q)_{m}$ states with masses $M_{B_{n}}=\Delta_{n}+m_{Q}$ and transition potentials $W_{n m}(r) \equiv V_{\bar{Q} Q \leftrightarrow \bar{B}_{n} B_{m}}(r)$ and which can be described by a coupled channel static Hamiltonian

$$
\mathcal{H}(r)=\left(\begin{array}{ccc}
V_{\bar{Q} Q}(r) & W(r) & \ldots \\
W(r) & 2 \Delta & \ldots \\
\vdots & \vdots & \ddots
\end{array}\right)
$$

and its corresponding eigenvalues $E_{n}^{\bar{Q} Q}(r)$. Some time ago Drummond suggested a string breaking and mixing scenarios on the lattice [18-20] where he proposed $W(r)=g e^{-m r}$ in the strong coupling 
limit. String breaking has been observed in lattice QCD by the SESAM Collaboration [21] for pion masses of $m_{\pi}=700 \mathrm{MeV}$ and using a linear combination of two modes $Q \bar{Q}$ and $B \bar{B}$ confirming a sharp avoided crossing pattern (see Fig. 2) at about $1.2 \mathrm{fm}$. In Fig. 2 we show the SESAM matrix elements of the coupled channel Hamiltonian of Eq. (6), where an almost constant $V_{\bar{B} B}(r)$ can be seen.

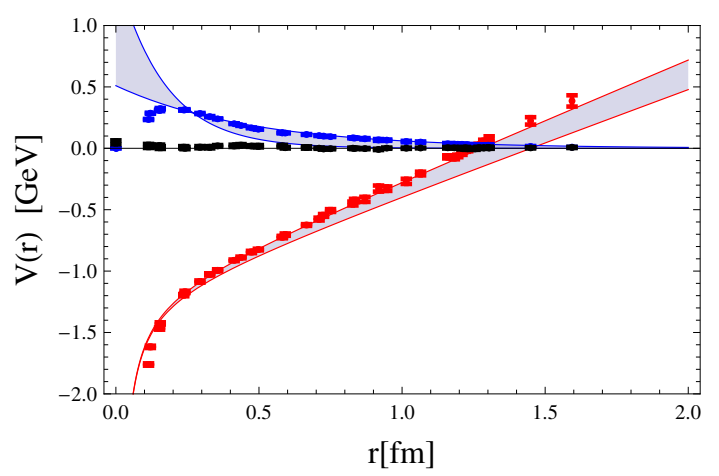

Figure 2. The coupled channel potential with the diagonal matrix elements $V_{\bar{Q} Q}(r)$ (red) and $V_{\bar{B} B}(r)$ (black) and the transition potential $V_{\bar{Q} Q \leftrightarrow \bar{B} B}(r)$ (blue). We compare our two-mode extraction [6] from finite temperature QCD free energies $F_{\bar{Q} Q}(r, T)$ with $N_{f}=2+1$ and physical pion mass [22] (gray bands), with the zero temperature determination and $m_{\pi}=700 \mathrm{MeV}[21]$ (error bars). To ease the comparison we shift the lowest energies at large distances to zero.

\section{Free energy and String breaking below the phase transition}

Relativistic heavy ions collisions have sufficient energy to produce heavy quarks in the fireball made of light $u, d, s$ quarks and gluons. On a more theoretical level, when we place two heavy colour conjugated sources, say $\bar{Q}$ and $Q$ at a fixed distance $r$ in the hot medium, we expect a thermodynamic shift of the equation of state, say $F_{\bar{Q} Q}(r, T)$, which is given in terms of a Polyakov loop correlator in the hot medium. In the static gauge we have $\Omega(\vec{r})=e^{i g A_{0}(\vec{r}) / T}$. The following dual relations hold [6]

$$
e^{-F_{\bar{Q} Q}(r, T)}=\langle\operatorname{tr} \Omega(r) \operatorname{tr} \Omega(0)\rangle_{T}=\frac{Z_{Q \otimes \bar{Q}}(r, T)}{Z_{0}(T)}=\frac{\sum_{n} e^{-E_{n}^{Q \otimes \Phi}(r) / T}}{\sum_{n} e^{-E_{n}^{0} / T}}=\sum_{n}|\langle n, T|\operatorname{tr} \Omega| 0, T\rangle|^{2} e^{-r w_{n}(T)},
$$

where the last equality is a Kallen-Lehmann-type representation which implies the new inequalities

$$
\partial_{r} F_{\bar{Q} Q}(r, T) \geq 0, \quad \partial_{r}^{2} F_{\bar{Q} Q}(r, T) \leq 0 .
$$

The asymptotics depends on the distance $r$ vs the thermal wavelength $1 / T$. For $r T \ll 1$ the hot medium becomes irrelevant, $F_{\bar{Q} Q}(r, T) \rightarrow V_{\bar{Q} Q}(r)$, whereas for $r T \gg 1$ cluster decomposition implies $F_{\bar{Q} Q}(r, T) \rightarrow F_{Q}(T)+F_{\bar{Q}}(T)$. Here $F_{Q}(T)=F_{\bar{Q}}(T)$ is the single quark free energy related to the expectation value of the Polyakov loop which becomes a partition function at low temperatures [23]

$$
\langle\operatorname{tr} \Omega\rangle_{T} \equiv e^{-F_{Q}(T) / T}=\frac{Z_{Q}}{Z_{0}} \approx \sum_{n} e^{-\Delta_{n} / T} .
$$

Our normalization is such that $\langle\operatorname{tr} \Omega\rangle_{T} \rightarrow N_{c}$ for $T \rightarrow \infty$. Below the phase transition the eigenvalues of the $\bar{Q} q$ Hamiltonian can be saturated with heavy-light mesonic states. Unfortunately, the number of such PDG states is too small [8] and we use as in [23] the corresponding RQM states [9, 10]. Likewise, the $\bar{Q} Q$ system can be saturated with the static Hamiltonian sketched in Eq. (6)

$$
e^{-F_{Q \bar{Q}}(r, T) / T} \approx \operatorname{Tr} e^{-\mathcal{H}(r) / T} .
$$



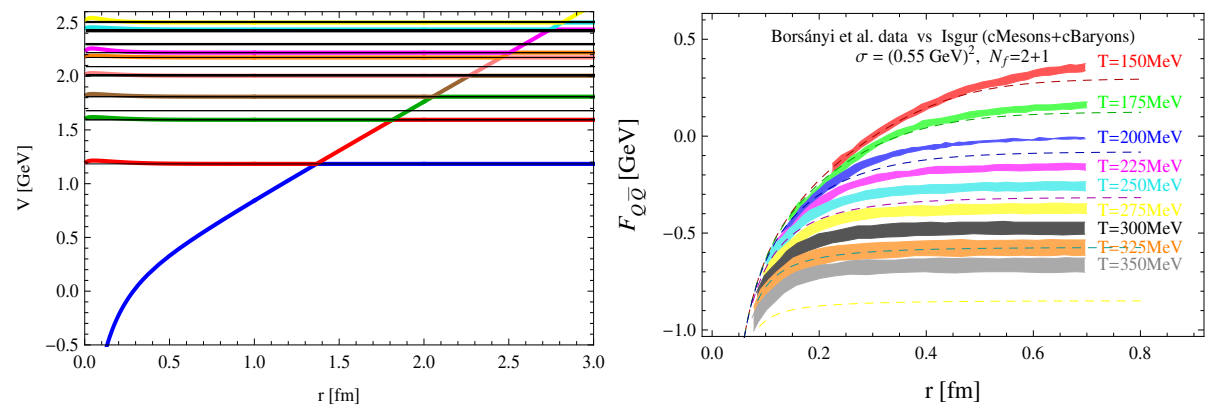

Figure 3. Avoided level crossing spectrum with multiple mixing as a function of distance with RQM using c-quark (left pane) extracted in [6] from the $\bar{Q} Q$ free energies obtained on the lattice [22] (right panel).

In Fig. 2 we compare our results for the two-mode Hamiltonian. From the heavy quark-antiquark free energy we extract a string breaking profile, $V_{\bar{Q} Q \leftrightarrow \bar{B} B}(r)=W(r)=g e^{-m r}$, conforming to Drummond's ideas [18-20]. A fit of the parameters of Eq. (5), with $c=0$ and $\Delta=0.472 \mathrm{GeV}$ gives

$$
\sqrt{\sigma}=0.424(14) \mathrm{GeV}, \quad g=0.98(47) \mathrm{GeV}, \quad m=0.80(38) \mathrm{GeV},
$$

with $r_{\text {corr }}(g, m)=0.98$. A complete avoided crossing pattern based on inclusion of more states with the same quantum numbers as $\bar{Q} Q, V_{\bar{Q} Q \leftrightarrow \bar{B}_{n} B_{m}}(r)$, only modifies the coupling but not the radial dependence and is shown in Fig. 3. We note that the lowest branch displays a more abrupt transition than assumed in (quenched) quark models [16] but similar to the generalized screened potential model [24, 25].

\section{Effective elementarity and coarse graining}

Within this mixing scenario both the heavy quarks as well as the heavy-light mesons $B$ and $\bar{B}$ are treated as pointlike, which they are not. The range of annihilation generated by the profile $W(r)=$ $g e^{-m r}$, is about $r_{s}=1 / m=0.25 \mathrm{fm}$, presumably smaller than the size of heavy-light mesons. This raises the question of what is the meaning of the interaction when they overlap. In order to elaborate on this, we will assume some underlying light-quark-string dynamics. Taking massless light quarks, $m_{q}=m_{\bar{q}}=0$, for simplicity we use the standard Salpeter Hamiltonian for the charge conjugated heavy-light meson-antimeson pairs $H_{q \bar{Q}}=p_{q}+\sigma r_{q}$ and $H_{\bar{q} Q}=p_{\bar{q}}+\sigma r_{\bar{q}}$. Thus, the spectrum is determined from the equation $(p+\sigma r) \Psi_{n}(r)=\Delta_{n} \Psi_{n}(r)$ whence the virial theorem relation yields $\Delta_{n}=2 \sigma\langle r\rangle_{n}$ [2]. This has the interesting interpretation that the string breaking happens at $\sigma r_{c}=$ $\Delta_{\bar{q} Q}+\Delta_{\bar{Q} q}$ corresponding to $r_{c}=2\left(\left\langle r_{q}\right\rangle+\left\langle r_{\bar{q}}\right\rangle\right)$, i.e. when the two extended mesons are about $t$ wice the overlapping distance. Therefore, $B$ and $\bar{B}$, are well separated and the overall separating distance $r$ is always well defined before they annihilate into the $\bar{Q} Q$ pair. This means that for $r>r_{c}$ the $\bar{Q} Q-\bar{B} B$ coupled channel dynamics can be treated as if all intervening particles were elementary.

We turn to the shape of the string breaking profile function. The transition model we have in mind from the $\bar{Q} Q$ to the $B \bar{B}$ is given by a contact interaction close in spirit to the ${ }^{3} P_{0}$-model $v_{0 \rightarrow q \bar{q}}\left(\vec{r}_{q}-\vec{r}_{\bar{q}}\right)=$ $g \delta^{(3)}\left(\vec{r}_{q}-\vec{r}_{\bar{q}}\right)$. In reality, this function will be smeared and, to see how, let us work in perturbation theory, where $\Psi_{n, m}\left(r_{q}, r_{\bar{q}}\right)=\Psi_{B, n}\left(r_{\bar{q}}\right) \Psi_{\bar{B}, m}\left(r_{q}\right)$ and $\Delta_{n m}=\Delta_{n}+\Delta_{m}$. Then the transition matrix element for two mesons located at a relative distance $r$ is given by

$$
W_{n, m}(r)=\int d^{3} r_{q} d^{3} r_{\bar{q}} \Psi_{B, n}\left(r_{\bar{q}}+r / 2\right) \Psi_{\bar{B}, m}\left(r_{q}-r / 2\right) v_{0 \rightarrow q \bar{q}}\left(r_{q}-r_{\bar{q}}\right) .
$$


This folding structure yields the profile function in momentum space,

$$
W(p)=\Psi_{0}^{2}(p) v_{0 \rightarrow \bar{q} q}(p)=W(0) \frac{m^{4}}{\left(m^{2}+p^{2}\right)^{2}} .
$$

The very short range of the transition potential together with the effective elementarity of the heavylight mesons suggests a coarse graining in the interaction, provided the typical wavelengths are longer than $r_{s}=1 / \mathrm{m}$. That this is the case can be seen in the nodal structure of $\bar{Q} Q$ quark model wave functions up to $n=5$ [16]. This is very similar to the pattern described by van Beveren and Rupp [26] where they take $W(r)=G \delta\left(r-r_{s}\right)$ and for which they find $r_{s} \sim 0.4 \mathrm{fm}$, implying a longer high momentum tail since in their case $W(p)=W(0) \sin \left(p r_{s}\right) /\left(p r_{s}\right)$. While the mass $m$ appearing in the transition potential was identified with pion exchange in the lattice calculation [21] for pion masses of $m_{\pi}=700 \mathrm{MeV}$, our results, extracted for physical $m_{\pi}$ free energies [22] and phenomenology, suggest that this is not the case. A more complete analysis will be presented elsewhere.

\section{References}

[1] K. Huang, Statistical Mechanics (Addison Wesley \& Sons, New York, 1987)

[2] E. Ruiz Arriola, L.L. Salcedo, E. Megias, Acta Phys. Polon. B45, 2407 (2014), 1410 3869

[3] E. Ruiz Arriola, L.L. Salcedo, E. Megias, Acta Phys. Polon. Supp. 8, 439 (2015), 1505.02922

[4] E. Megias, E. Ruiz Arriola, L.L. Salcedo, Nucl. Part. Phys. Proc. 93-97, 270 (2015), [Nucl. Part. Phys. Proc.270-272,170(2016)], 1507.08606

[5] E. Megias, E. Ruiz Arriola, L.L. Salcedo, Acta Phys. Polon. Supp. 9, 401 (2016), 1605. 04453

[6] E. Megias, E. Ruiz Arriola, L.L. Salcedo (2016), 1603.04642

[7] E.R. Arriola, W. Broniowski, P. Masjuan, Acta Phys.Polon.Supp. 6, 95 (2013), 1210. 7153

[8] C. Patrignani, Chin. Phys. C40, 100001 (2016)

[9] S. Godfrey, N. Isgur, Phys.Rev. D32, 189 (1985)

[10] S. Capstick, N. Isgur, Phys.Rev. D34, 2809 (1986)

[11] W. Florkowski, Phenomenology of Ultra-Relativistic Heavy-Ion Collisions (World Scientific, Singapore, 2010)

[12] Y. Aoki, G. Endrodi, Z. Fodor, S. Katz, K. Szabo, Nature 443, 675 (2006), hep-lat/0611014

[13] S. Borsanyi, Z. Fodor, C. Hoelbling, S.D. Katz, S. Krieg et al., Phys.Lett. B730, 99 (2014)

[14] A. Bazavov et al. (HotQCD Collaboration) (2014), 1407.6387

[15] S. Necco, R. Sommer, Nucl. Phys. B622, 328 (2002), hep-lat/0108008

[16] J. Segovia, D.R. Entem, F. Fernandez, E. Ruiz Arriola, Phys. Rev. D85, 074001 (2012)

[17] J. Ferretti, E. Santopinto, Phys. Rev. D90, 094022 (2014), 1306. 2874

[18] I.T. Drummond, Phys. Lett. B434, 92 (1998), hep-lat/9805012

[19] I.T. Drummond, Phys. Lett. B442, 279 (1998), hep-lat/9808014

[20] I.T. Drummond, R.R. Horgan, Phys. Lett. B447, 298 (1999), hep-lat/9811016

[21] G.S. Bali, H. Neff, T. Duessel, T. Lippert, K. Schilling (SESAM Collaboration), Phys.Rev. D71, 114513 (2005), hep-lat/0505012

[22] S. Borsányi, Z. Fodor, S.D. Katz, A. Pásztor, K.K. Szabó, C. Török, JHEP 04, 138 (2015)

[23] E. Megias, E. Ruiz Arriola, L. Salcedo, Phys.Rev.Lett. 109, 151601 (2012), 1204.2424

[24] P. Gonzalez, J. Phys. G41, 095001 (2014), 1406. 5025

[25] P. González, Phys. Rev. D92, 014017 (2015), 1507 . 02397

[26] E. van Beveren, G. Rupp, Annals Phys. 324, 1620 (2009), 0809. 1149 